\title{
Low daily salt intake is correlated with albuminuria in patients with type 2 diabetes
}

\author{
Kazumi Sakabe ${ }^{1}$, Michiaki Fukui ${ }^{1}$, Emi Ushigome ${ }^{1}$, Masahide Hamaguchi ${ }^{2}$, Takafumi Senmaru ${ }^{1}$, \\ Masahiro Yamazaki ${ }^{1}$, Goji Hasegawa ${ }^{1}$ and Naoto Nakamura ${ }^{1}$
}

Both high and low salt intakes have been reported to be associated with an increased risk of cardiovascular events. The aim of this study was to investigate the relationship between daily salt intake and albuminuria, a marker of diabetic nephropathy and cardiovascular disease, in patients with type 2 diabetes. We classified 270 patients with type 2 diabetes, who were not receiving antihypertensive medication into four groups according to their daily salt intake $(<8,8-10,10-12$ and $>12 \mathrm{~g}$ per day). We investigated the relationship between daily salt intake and the logarithm of urinary albumin excretion (UAE). A multivariate linear regression analysis was used to evaluate whether daily salt intake independently correlated with the logarithm of UAE. In addition, we assessed the contribution of the variables, including age, sex, duration of diabetes, body mass index, systolic blood pressure, hemoglobin $A_{1 C}$, low-density lipoprotein cholesterol, triglycerides, serum creatinine, alcohol intake, smoking status and square of (daily salt intake -10) on albuminuria, defined as a UAE $>30 \mathrm{mgg}^{-1}$ of creatinine, using a multiple logistic regression analysis. The logarithm of the UAE was lowest in the third quartile of daily salt intake. The multivariate linear regression analysis demonstrated that the logarithm of the UAE was significantly correlated with the quadratic term of daily salt intake centered at $10 \mathrm{~g}$ per day $(\beta=0.170, P=0.008)$. The multivariate logistic regression analysis demonstrated that the odds ratio $(95 \%$ confidence interval) of albuminuria was $3.996(1.295-12.327 ; P=0.016)$ in patients whose daily salt intake was less than $8 \mathrm{~g}$ per day compared with patients whose daily salt intake was $10-12 \mathrm{~g}$ per day. In conclusion, low daily salt intake was correlated with albuminuria in patients with type 2 diabetes, who were not receiving antihypertensive medication.

Hypertension Research (2012) 35, 1176-1179; doi:10.1038/hr.2012.116; published online 26 July 2012

Keywords: blood pressure; daily salt intake; diabetic nephropathy

\section{INTRODUCTION}

The purpose of long-term care for diabetic patients is to prevent the development of diabetic complications. Both diabetes mellitus and hypertension are important risk factors for diabetic nephropathy and cardiovascular disease. Therefore, it is important to control both blood pressure and blood glucose to prevent microvascular and macrovascular complications.

Epidemiological and clinical studies have demonstrated that high salt intake is associated with hypertension and an increased risk of cardiovascular events. ${ }^{1-4}$ High salt intake is a predictor of mortality and risk of coronary heart disease independent of other cardiovascular risk factors, including blood pressure. ${ }^{5}$ Furthermore, high salt intake increases extracellular sodium concentrations, may adversely affect vascular reactivity, ${ }^{6}$ and induces myocardial and renal fibrosis. $^{7}$ On the basis of these results, many guidelines have recommended salt restriction, especially for patients with both type 2 diabetes mellitus and hypertension. Recently, however, low salt intake has also been reported to be associated with an increased risk of cardiovascular events and mortality. ${ }^{8-10}$ In this study, we investigated the correlation between daily salt intake and albuminuria in patients with type 2 diabetes.

\begin{abstract}
METHODS
Patients

Daily salt intake was measured in 270 consecutive patients with type 2 diabetes, who were not receiving antihypertensive medications and who had regularly attended diabetes outpatient clinics at the Hospital of Kyoto Prefectural University of Medicine or one of four other general hospitals. Blood pressure was measured three times in one session by a trained nurse using an automatic device, the HEM-70801C (Omron Healthcare Co. Ltd, Kyoto, Japan), with the patient seated after resting for at least $5 \mathrm{~min}$. Type 2 diabetes was diagnosed according to the Report of the Expert Committee on the Diagnosis and Classification of Diabetes Mellitus. ${ }^{11}$ Retinopathy was assessed using mydriasis by ophthalmologists who were unaware of the data of both patient daily salt
\end{abstract}

${ }^{1}$ Department of Endocrinology and Metabolism, Kyoto Prefectural University of Medicine, Graduate School of Medical Science, Kamigyo-ku, Kyoto, Japan and ${ }^{2}$ Laboratory of Experimental Immunology, WPI Immunology Frontier Research Center, Osaka University, Suita, Japan

Correspondence: Dr M Fukui, Department of Endocrinology and Metabolism, Kyoto Prefectural University of Medicine, Graduate School of Medical Science, 465 Kajii-cho, Kawaramachi-Hirokoji, Kamigyo-ku, Kyoto 602-8566, Japan.

E-mail: sayarinapm@hotmail.com

Received 9 April 2012; revised 12 May 2012; accepted 17 May 2012; published online 26 July 2012 
intake and blood pressure, and was graded using the following categories: no diabetic retinopathy, simple diabetic retinopathy and proliferative (including preproliferative) diabetic retinopathy. If the findings in the left and right fundi were discordant, the worse side was taken as representative for the subject. Cardiovascular disease was defined as a previous myocardial or cerebral infarction based on the clinical history or physical examination. We excluded patients who had advanced renal dysfunction (serum creatinine $>2.0 \mathrm{mg} \mathrm{dl}^{-1}$ ). Information, including age, duration of diabetes, microvascular complications, macrovascular complications, alcohol intake and smoking status, was obtained at the time of blood pressure measurement. Alcohol intake (never, social or daily) and smoking status (never, past or current) were assessed by interview. All patients gave their informed consent to participate in the study.

\section{Study design}

We classified 270 patients with type 2 diabetes, who were not receiving antihypertensive medication into four groups according to daily salt intake $(<8,8-10,10-12$ and $>12 \mathrm{~g}$ per day). We investigated the relationship between daily salt intake and the logarithm of urinary albumin excretion (UAE). We then evaluated if daily salt intake independently correlated with the logarithm of UAE by multivariate linear regression analysis after adjustment for the following variables as confounding factors: age, sex, duration of diabetes, body mass index, systolic blood pressure, hemoglobin $A_{1 C}$, lowdensity lipoprotein cholesterol, triglycerides, serum creatinine, alcohol intake, smoking status and daily salt intake. In addition, we assessed the contribution of the variables on albuminuria, defined as $\mathrm{UAE}>30 \mathrm{mgg}^{-1}$ of creatinine, using a multiple logistic regression analysis. Approval for the study was obtained from the local Research Ethics Committee, and informed consent was obtained from all participants. This study was conducted in accordance with Declaration of Helsinki.

\section{Data collection}

Blood samples for biochemical measurements were taken in the morning. Hemoglobin $A_{1 C}$ (expressed as National Glycohemoglobin Standardization Program values), serum lipid concentrations and other biochemical data were determined by standard laboratory measurements. The UAE was measured with an immunoturbidimetric assay. A mean value for the UAE was determined from three urine collections. The glomerular filtration rate was estimated using the equation of the Japanese Society of Nephrology: estimated glomerular filtration rate $=194 \times \mathrm{Cr}^{-1.094} \times \mathrm{age}^{-0.287}\left(\mathrm{ml} \mathrm{min}^{-1}\right.$ per $1.73 \mathrm{~m}^{2}$ ). For women, the estimated glomerular filtration rate was multiplied by a correction factor of 0.739 . Daily salt intake was estimated by a spot urine sample using the following equation: $0.0585 \times 21.98 \times$ (urinary sodium/ urinary creatinine $\times(14.89 \times$ body weight $(\mathrm{kg})+16.14 \times$ height $(\mathrm{cm})-2.04 \times$ age -2244.45$))^{0.392}{ }^{12}$

\section{Statistical analysis}

The values are expressed as the means \pm s.d. for the continuous variables and as the number of patients for the categorical variables. As the UAE showed a skewed distribution, the logarithmic transformation was carried out before performing the statistical analysis. A one-way analysis of variance was performed to detect differences among the four groups. The $\chi^{2}$-test was used to compare categorical variables among the four groups. In the multivariate analysis, the independent variables included age, sex, duration of diabetes, body mass index, systolic blood pressure, hemoglobin $A_{1 C}$, low-density lipoprotein cholesterol, triglycerides, serum creatinine, alcohol intake, smoking status and square of (daily salt intake-10). We designed a multivariate linear regression model including a quadratic term of daily salt intake to identify factors independently correlated with the logarithm of UAE because we assumed a reverse J-shaped relationship rather than a linear relationship between daily salt intake and the logarithm of UAE. In addition, we assessed the contribution of the variables on albuminuria, defined as $\mathrm{UAE}>30 \mathrm{mg} \mathrm{g}^{-1}$ creatinine, using a multiple logistic regression analysis. Values of $P<0.05$ were considered statistically significant. All statistical analyses were performed using SPSS version 11.0 J (SPSS Inc., Chicago, IL, USA).

\section{RESULTS}

The mean daily salt intake was $8.8 \pm 2.3 \mathrm{~g}$ per day. The clinical characteristics of the patients according to the quartiles of daily salt intake are shown in Table 1. There was a significant increasing trend in body mass index and degree of retinopathy with increasing daily salt intake. Conversely, there was a significant decreasing trend in age

Table 1 Characteristics of patients

\begin{tabular}{|c|c|c|c|c|c|}
\hline & \multicolumn{4}{|c|}{ Quartile of daily salt intake } & \multirow[b]{2}{*}{ P-values } \\
\hline & 1 & 2 & 3 & 4 & \\
\hline$n$ & 96 & 101 & 51 & 22 & \\
\hline Sex (male/female) & $42 / 54$ & $55 / 46$ & $28 / 23$ & $10 / 12$ & 0.396 \\
\hline Age (years) & $65.1 \pm 10.8$ & $64.9 \pm 9.4$ & $61.0 \pm 10.0$ & $56.3 \pm 10.9$ & 0.001 \\
\hline Duration of diabetes (years) & $12.6 \pm 10.2$ & $13.5 \pm 10.4$ & $10.1 \pm 8.3$ & $11.0 \pm 9.0$ & 0.222 \\
\hline Body mass index $\left(\mathrm{kg} \mathrm{m}^{-2}\right)$ & $22.4 \pm 3.7$ & $22.9 \pm 3.3$ & $23.1 \pm 3.5$ & $24.9 \pm 3.6$ & 0.028 \\
\hline Systolic blood pressure (mm Hg) & $137.1 \pm 21.1$ & $139.2 \pm 18.7$ & $134.6 \pm 15.8$ & $131.8 \pm 13.3$ & 0.268 \\
\hline Diastolic blood pressure (mm Hg) & $76.5 \pm 12.3$ & $76.1 \pm 11.0$ & $77.9 \pm 11.8$ & $79.0 \pm 8.2$ & 0.633 \\
\hline Hemoglobin $A_{1 C}(\%)$ & $7.2 \pm 0.9$ & $7.1 \pm 0.7$ & $7.3 \pm 1.0$ & $7.6 \pm 1.0$ & 0.186 \\
\hline LDL cholesterol $\left(\mathrm{mmoll}^{-1}\right)$ & $2.7 \pm 0.8$ & $2.8 \pm 0.7$ & $2.8 \pm 0.6$ & $3.0 \pm 0.7$ & 0.656 \\
\hline Triglycerides $\left(\mathrm{mmol} \mathrm{l}^{-1}\right)$ & $3.3 \pm 1.9$ & $3.0 \pm 1.6$ & $3.6 \pm 2.4$ & $3.2 \pm 1.8$ & 0.409 \\
\hline Creatinine (mg dl ${ }^{-1}$ ) & $0.70 \pm 0.18$ & $0.72 \pm 0.18$ & $0.72 \pm 0.19$ & $0.68 \pm 0.20$ & 0.701 \\
\hline Estimated GFR ( $\mathrm{ml} \mathrm{min}{ }^{-1}$ per $\left.1.73 \mathrm{~m}^{2}\right)$ & $78.4 \pm 18.7$ & $78.7 \pm 19.8$ & $79.0 \pm 17.1$ & $84.5 \pm 19.5$ & 0.594 \\
\hline Retinopathy (NDR/SDR/PDR) & $77 / 11 / 8$ & $75 / 14 / 11$ & $41 / 8 / 2$ & $11 / 3 / 8$ & 0.015 \\
\hline Cardiovascular disease $(-/+)$ & $83 / 13$ & $84 / 17$ & $48 / 3$ & $21 / 1$ & 0.164 \\
\hline Alcohol (never/social/daily) & $61 / 15 / 19$ & $58 / 26 / 17$ & $25 / 15 / 11$ & $12 / 7 / 3$ & 0.264 \\
\hline Smoking (never/past/current) & $53 / 28 / 15$ & $57 / 24 / 20$ & $25 / 13 / 13$ & $15 / 5 / 2$ & 0.556 \\
\hline $\log (\mathrm{UAE})$ & $1.39 \pm 0.55$ & $1.37 \pm 0.53$ & $1.09 \pm 0.43$ & $1.23 \pm 0.48$ & 0.004 \\
\hline
\end{tabular}

Abbreviations: GFR, glomerular filtration rate; LDL, low-density lipoprotein; NDR, no diabetic retinopathy; PDR, proliferative diabetic retinopathy; SDR, simple diabetic retinopathy; UAE, urinary albumin excretion. Date are mean \pm s.d. or number of patients. One-way analysis of variance was performed to detect differences among the four groups; $\chi^{2}$-test was used to compare categorical variables among the

${ }^{a}$ Cut points for quartiles of daily salt intake are 8,10 and $12 \mathrm{~g}$ per day. 
with increasing daily salt intake. A box and whisker plot was used to demonstrate the correlation between daily salt intake and the logarithm of UAE, and showed that the logarithm of UAE in the third quartile of daily salt intake was significantly lower than that in the first $(P<0.01)$ or second $(P<0.05)$ quartile of daily salt intake (Figure 1).

In the multivariate linear regression analysis, which included a quadratic term of daily salt intake centered at $10 \mathrm{~g}$ per day, the logarithm of UAE was independently correlated with age, systolic blood pressure, smoking status and the quadratic term of daily salt intake centered at $10 \mathrm{~g}$ per day (Table 2). The multivariate logistic regression analysis demonstrated that the odds ratio (95\% confidence interval) for albuminuria was significantly higher in patients whose daily salt intake was less than $8 \mathrm{~g}$ per day compared with that in patients whose daily salt intake was $10-12 \mathrm{~g}$ per day (Table 3 ). In addition, age, systolic blood pressure, low-density lipoprotein cholesterol and serum creatinine independently correlated with albuminuria.

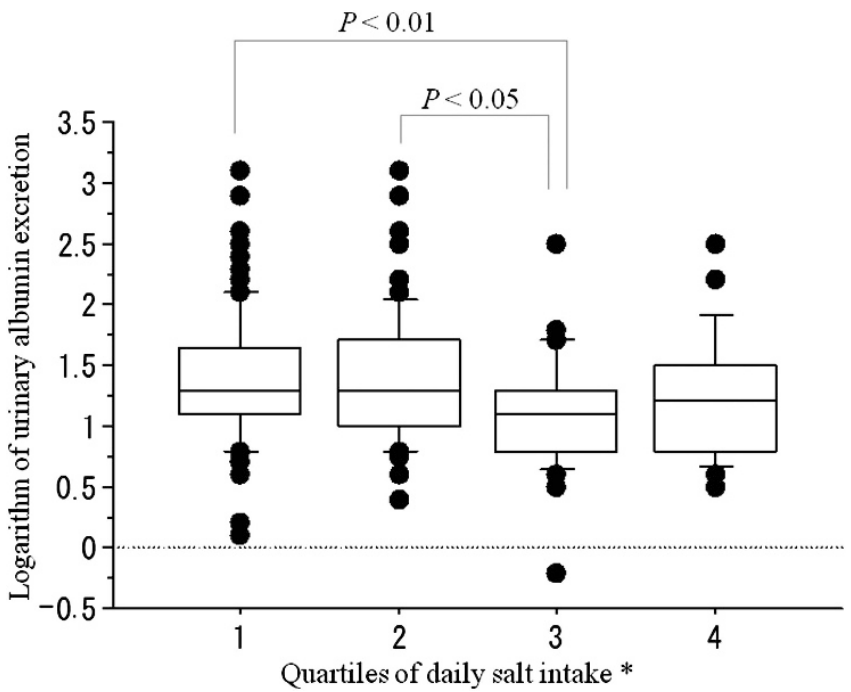

Figure 1 Correlation between the quartiles of daily salt intake and the logarithm of urinary albumin excretion. Data are presented as medians, 25th and 75th percentiles (boxes), and 10th and 90th percentiles (whiskers). The logarithm of urinary albumin excretion in the third quartile was significantly lower than in the first $(P<0.01)$ or second $(P<0.05)$ quartile of daily salt intake. ${ }^{*}$ Cut points for the quartiles of daily salt intake were 8,10 and $12 \mathrm{~g}$.

Table 2 Multivariate linear regression analysis on logarithm of UAE

\begin{tabular}{lrr}
\hline & $\beta$ & P-values \\
\hline Sex & -0.159 & 0.087 \\
Age & 0.172 & 0.021 \\
Duration of diabetes & 0.046 & 0.493 \\
Body mass index & 0.036 & 0.629 \\
Systolic blood pressure & 0.226 & $<0.001$ \\
Hemoglobin A 1 c & 0.055 & 0.412 \\
LDL cholesterol & -0.103 & 0.118 \\
Triglycerides & -0.045 & 0.497 \\
Creatinine & 0.120 & 0.147 \\
Alcohol & 0.013 & 0.845 \\
Smoking & 0.244 & 0.002 \\
(Daily salt intake -10$)^{2}$ & 0.170 & 0.008 \\
\hline
\end{tabular}

Abbreviations: LDL, Iow-density lipoprotein; UAE, urinary albumin excretion.

\section{DISCUSSION}

This study revealed a reverse J-shaped relationship between daily salt intake and albuminuria in patients with type 2 diabetes. Furthermore, a quadratic term of daily salt intake centered at $10 \mathrm{~g}$ per day correlated with the logarithm of UAE independent of classical risk factors. No previous study included a low salt-intake group, or applied a quadratic term of daily salt intake in a linear regression model. The use of these methodologies may have led to our new findings.

Previous studies have demonstrated that high salt intake is associated with an increased risk of cardiovascular events. ${ }^{1-3}$ However, recent studies have reported the possibility that low salt intake might also be associated with an increased risk of cardiovascular events and mortality. In patients with type 2 diabetes, low 24-h urinary sodium excretion has paradoxically been associated with increased all-cause and cardiovascular mortality. ${ }^{8-10}$ Concerns about low-salt diets were first raised by Meneely et al..$^{13}$ and Dahl and Love, ${ }^{14}$ who linked decreased salt intake to growth failure and increased mortality in rats. Dong et al. ${ }^{15}$ revealed that low dietary salt intake independently predicts the high overall and cardiovascular mortality of dialysis patients.

One possible explanation of this result is that excessive dietary salt restriction leads to increased angiotensin II and aldosterone levels, chiefly via an increase in plasma renin activity. There is also evidence that a reduced salt intake is associated with the activation of metabolic and neurohormonal pathways, including the sympathetic nervous system and the renin-angiotensin-aldosterone system, an increase in total and low-density lipoprotein cholesterol and a reduction of peripheral insulin sensitivity. ${ }^{16-18}$

Our study has several limitations. First, the cause and effect relationship between daily salt intake and the logarithm of UAE could not be determined because this study is cross-sectional. Second, daily salt intake was estimated from a spot urine sample. However, the

Table 3 Multivariate adjusted odds ratio for albuminuria in patients with type 2 diabetes

\begin{tabular}{|c|c|c|}
\hline & Odds ratio $(95 \% \mathrm{Cl})$ & P-values \\
\hline \multicolumn{3}{|l|}{ Daily salt intake } \\
\hline Quartile 1 & $3.996(1.295-12.327)$ & 0.016 \\
\hline Quartile 2 & 3.052 (1.001-9.304) & 0.050 \\
\hline Quartile 3 & 1 & \\
\hline Quartile 4 & $2.674(0.540-13.248)$ & 0.228 \\
\hline Sex $($ female $=0$, male $=1)$ & $0.536(0.183-1.572)$ & 0.256 \\
\hline Age & $1.050(1.005-1.096)$ & 0.028 \\
\hline Duration of diabetes & $1.011(0.977-1.046)$ & 0.540 \\
\hline Body mass index & $1.030(0.918-1.155)$ & 0.619 \\
\hline Systolic blood pressure & $1.034(1.015-1.054)$ & $<0.001$ \\
\hline Hemoglobin $A_{1 C}$ & $1.033(0.668-1.597)$ & 0.883 \\
\hline LDL cholesterol & $0.985(0.972-0.998)$ & 0.027 \\
\hline Triglycerides & $0.999(0.993-1.004)$ & 0.614 \\
\hline Creatinine ( $0.1 \mathrm{mg} \mathrm{dl}^{-1}$ increase) & $1.291(1.019-1.637)$ & 0.035 \\
\hline \multicolumn{3}{|l|}{ Alcohol } \\
\hline Never & 1 & \\
\hline Social & $0.586(0.234-1.464)$ & 0.253 \\
\hline Daily & $1.005(0.392-2.575)$ & 0.992 \\
\hline \multicolumn{3}{|l|}{ Smoking } \\
\hline None & 1 & \\
\hline Past & $1.304(0.462-3.682)$ & 0.617 \\
\hline Current & $2.350(0.785-7.041)$ & 0.127 \\
\hline
\end{tabular}

Abbreviations: $\mathrm{Cl}$, confidence interval; LDL, low-density lipoprotein. 
values obtained from the spot urine method correlated highly with those obtained from 24-h urinary sodium excretion, ${ }^{19}$ and the usefulness of the spot urine method has been confirmed in population studies $^{20}$ and in hypertensive patients. ${ }^{21}$ We speculate that excessive dietary salt restriction leads to increased angiotensin II and aldosterone levels, chiefly via an increase in plasma renin activity. Protein consumption is also known to be a critical factor in albuminuria. Unfortunately, we do not have those data in our study.

Recent reports, including the present study, raise the possibility that a strict restriction in dietary salt intake may be a risk factor for cardiovascular disease. This finding requires further investigation with well-designed, large-scale prospective studies.

\section{CONCLUSIONS}

Low daily salt intake was correlated with albuminuria in patients with type 2 diabetes, who were not receiving antihypertensive medication.

\section{CONFLICT OF INTEREST}

The authors declare no conflict of interest.

\section{ACKNOWLEDGEMENTS}

We thank Dr Shinobu Inada at Kyoto Prefectural University of Medicine; Drs Atsushi Omoto, Toru Tanaka and Wataru Fukuda at Kyoto First Red Cross Hospital; Dr Haruhiko Atsuta at Kyoto Second Red Cross Hospital; Drs Masayoshi Ohnishi, Shin-ichi Mogami and Yoshihiro Kitagawa at Osaka General Hospital of West Japan Railway Company; and Drs Sei Tsunoda and Yohei Oda at Social Insurance Kyoto Hospital for their assistance in researching the data. We also thank Naoko Higo, Machiko Hasegawa and Terumi Kaneko at Kyoto Prefectural University of Medicine for measuring blood pressure and Sayoko Horibe, Hiroko Kawamura and Sanae Utena at Kyoto Prefectural University of Medicine for their secretarial assistance.

Author contributions: KS researched the data and wrote the manuscript; MF, $\mathrm{EU}$ and GH researched the data and contributed to the discussion; $\mathrm{MH}$, TS and MY contributed to the discussion; and NN reviewed and edited the manuscript.

1 He FJ, MacGregor GA. How far should salt intake be reduced? Hypertension 2003; 42 1093-1099.

2 Umesawa M, Iso H, Date C, Yamamoto A, Toyoshima H, Watanabe Y, Kikuchi S, Koizumi A, Kondo T, Inaba Y, Tanabe N, Tamakoshi A. JACC Study Group. Relations between dietary sodium and potassium intakes and mortality from cardiovascular disease: the Japan Collaborative Cohort Study for Evaluation of Cancer Risks. Am J Clin Nutr 2008; 88: 195-202.

3 He J, Ogden LG, Vupputuri S, Bazzano LA, Loria C, Whelton PK. Dietary sodium intake and subsequent risk of cardiovascular disease in overweight adults. JAMA 1999; 282: 2027-2034

4 Mohan S, Campbell NR. Salt and high blood pressure. Clin Sci 2009; 117: 1-11.

5 Tuomilehto J, Jousilahti P, Rastenyte D, Moltchanov V, Tanskanen A, Pietinen P, Nissinen A. Urinary sodium excretion and cardiovascular mortality in Finland: a prospective study. Lancet 2001; 357: 848-851.

6 Simon G. Experimental evidence for blood pressure-independent vascular effects of high sodium diet. Am J Hypertens 2003; 16: 1074-1078.

7 Yu HC, Burrell LM, Black MJ, Wu LL, Dilley RJ, Cooper ME, Johnston CI. Salt induces myocardial and renal fibrosis in normotensive and hypertensive rats. Circulation 1998; 98: 2621-2628.

8 Ekinci El, Clarke S, Thomas MC, Moran JL, Cheong K, Maclsaac RJ, Jerums G. Dietary salt intake and mortality in patients with type 2 diabetes. Diabetes Care 2011; 34: 703-709.

9 Alderman $\mathrm{MH}$, Madhavan S, Cohen $\mathrm{H}$, Sealey JE, Laragh JH. Low urinary sodium is associated with greater risk of myocardial infarction among treated hypertensive men. Hypertension 1995; 25: 1144-1152.

10 Cohen HW, Hailpern SM, Alderman MH. Sodium intake and mortality follow-up in the Third National Health and Nutrition Examination Survey (NHANES III). J Gen Intern Med 2008; 23: 1297-1302.

11 The Expert Committee on the Diagnosis and Classification of Diabetes Mellitus. Report of the expert committee on the diagnosis and classification of diabetes mellitus. Diabetes Care 2003; 26: S5-S20.

12 Kawano Y, Tsuchihashi T, Matsuura H, Ando K, Fujita T, Ueshima H. Working Group for Dietary Salt Reduction of the Japanese Society of Hypertension. Report of the Working Group for Dietary Salt Reduction of the Japanese Society of Hypertension: (2) Assessment of salt intake in the management of hypertension. Hypertens Res 2007; 30: 887-893.

13 Meneely GR, Ball COT, Youmans JB. Chronic sodium chloride toxicity: the protective effect of added potassium chloride. Ann Intern Med 1957; 47: 263-273.

14 Dahl LK, Love RM. Evidence for relationship between sodium (chloride) intake and human hypertension. Arch Intern Med 1954; 94: 525-531.

15 Dong J, Li Y, Yang Z, Luo J. Low dietary sodium intake increases the death risk in peritoneal dialysis. Clin J Am Soc Nephrol 2010; 5: 240-247.

16 Graudal NA, Galløe AM, Garred P. Effects of sodium restriction on blood pressure, renin, aldosterone, catecholamines, cholesterols, and triglyceride: a meta-analysis. JAMA 1998; 279: 1383-1391.

17 Petrie JR, Morris AD, Minamisawa K, Hilditch TE, Elliott HL, Small M, McConnell J. Dietary sodium restriction impairs insulin sensitivity in noninsulin-dependent diabetes mellitus. J Clin Endocrinol Metab 1998; 83: 1552-1557.

18 Grassi G, Cattaneo BM, Seravalle G, Lanfranchi A, Bolla G, Mancia G. Baroreflex impairment by low sodium diet in mild or moderate essential hypertension. Hypertension 1997: 29: 802-807.

19 Kawasaki T, Itoh K, Uezono K, Sasaki H. A simple method for estimating $24 \mathrm{~h}$ urinary sodium and potassium excretion from second morning voiding urine specimen in adults. Clin Exp Pharmacol Physiol 1993; 20: 7-14.

20 Iseki K, Iseki C, Itoh K, Uezono K, Sanefuji M, Ikemiya Y, Fukiyama K, Kawasaki T. Urinary excretion of sodium and potassium in a screened cohort in Okinawa, Japan. Hypertens Res 2002; 25: 731-736.

21 Kawamura M, Kusano Y, Takahashi T, Owada M, Sugawara T. Effectiveness of a spot urine method in evaluating daily salt intake in hypertensive patients taking oral antihypertensive drugs. Hypertens Res 2006; 29: 397-402. 